\title{
TWO UPPER LIMITS ON THE ROSSITER-MCLAUGHLIN EFFECT, WITH DIFFERING IMPLICATIONS: WASP-1 HAS A HIGH OBLIQUITY AND WASP-2 IS INDETERMINATE*
}

\author{
Simon Albrecht ${ }^{1}$, Joshua N. Winn ${ }^{1}$, John Asher Johnson ${ }^{2,3}$, R. Paul Butler ${ }^{4}$, Jeffreey D. Crane ${ }^{5}$, \\ Stephen A. Shectman ${ }^{5}$, Ian B. Thompson ${ }^{5}$, Norio Narita ${ }^{6}$, Bun'ei Sato $^{7}$, Teruyuki Hirano ${ }^{1,8}, \mathrm{Keigo} \mathrm{Enya}^{9}$, \\ AND DEBRA Fischer ${ }^{10}$ \\ ${ }^{1}$ Department of Physics, and Kavli Institute for Astrophysics and Space Research, Massachusetts Institute of Technology, Cambridge, MA 02139, USA \\ ${ }^{2}$ California Institute of Technology, Department of Astrophysics, MC249-17, Pasadena, CA 91125, USA \\ ${ }^{3}$ NASA Exoplanet Science Institute (NExScI), California Institute of Technology, Pasadena, CA 91125, USA \\ ${ }^{4}$ Department of Terrestrial Magnetism, Carnegie Institution of Washington, 5241 Broad Branch Road NW, Washington, DC 20015, USA \\ ${ }^{5}$ The Observatories of the Carnegie Institution of Washington, 813 Santa Barbara Street, Pasadena, CA 91101, USA \\ ${ }^{6}$ National Astronomical Observatory of Japan, 2-21-1 Osawa, Mitaka, Tokyo 181-8588, Japan \\ ${ }^{7}$ Department of Earth and Planetary Sciences, Graduate School of Science and Engineering, Tokyo Institute of Technology, 2-12-1 Ookayama, \\ Meguro-ku, Tokyo 152-8551, Japan \\ ${ }^{8}$ Department of Physics, The University of Tokyo, Tokyo 113-0033, Japan \\ ${ }^{9}$ Department of Infrared Astrophysics, Institute of Space and Astronautical Science, Japan Aerospace Exploration Agency, 3-1-1 Yoshinodai, \\ Chuo-ku, Sagamihara, Kanagawa 252-5210, Japan \\ ${ }^{10}$ Department of Astronomy, Yale University, New Haven, CT 06511, USA \\ Received 2011 May 19; accepted 2011 June 10; published 2011 August 11
}

\begin{abstract}
We present precise radial-velocity (RV) measurements of WASP-1 and WASP-2 throughout transits of their giant planets. Our goal was to detect the Rossiter-McLaughlin (RM) effect, the anomalous RV observed during eclipses of rotating stars, which can be used to study the obliquities of planet-hosting stars. For WASP-1, a weak signal of a prograde orbit was detected with $\approx 2 \sigma$ confidence, and for WASP-2 no signal was detected. The resulting upper bounds on the RM amplitude have different implications for these two systems because of the contrasting transit geometries and the stellar types. Because WASP-1 is an F7V star, and such stars are typically rapid rotators, the most probable reason for the suppression of the RM effect is that the star is viewed nearly pole-on. This implies that the WASP-1 star has a high obliquity with respect to the edge-on planetary orbit. Because WASP-2 is a K1V star, and is expected to be a slow rotator, no firm conclusion can be drawn about the stellar obliquity. Our data and our analysis contradict an earlier claim that WASP-2b has a retrograde orbit, thereby revoking this system's status as an exception to the pattern that cool stars have low obliquities.
\end{abstract}

Key words: planetary systems - planets and satellites: formation - planet-star interactions - stars: rotation techniques: spectroscopic

Online-only material: color figures

\section{INTRODUCTION}

The existence of Jupiter-sized planets on very close-in orbits presents a challenge to any model which aims to explain the formation of planets. In the current picture, these planets form further away from their host star and migrate inward. How and why this migration occurs is subject to debate (e.g., Lin et al. 1996; Nagasawa et al. 2008). Recently, an important clue to this riddle was revealed: a subset of the close-in planets have orbits that are seeming randomly oriented with respect to the equatorial plane of the host star (see, e.g., Hébrard et al. 2008; Winn et al. 2009; Narita et al. 2009; Johnson et al. 2009; Triaud et al. 2010).

Winn et al. (2010) and Schlaufman (2010) found that planets orbiting stars with effective temperatures $\gtrsim 6250 \mathrm{~K}$ (i.e., mass $\gtrsim 1.2 M_{\odot}$ ) tend to have an orbital axis misaligned with respect to the stellar spin axis, i.e., a high stellar obliquity. In contrast, the two axes are generally well aligned for systems in which the host star is cooler (i.e., less massive). These authors noted that

\footnotetext{
* The data presented herein were collected with the Magellan (Clay) Telescope located at Las Campanas Observatory, Chile; the Subaru telescope, which is operated by the National Astronomical Observatory of Japan; and the Keck I telescope at the W. M. Keck Observatory, which is operated as a scientific partnership among the California Institute of Technology, the University of California and the National Aeronautics and Space Administration.
}

this could reflect a difference in the dominant planet migration mechanism between low-mass stars and high-mass stars. Winn et al. (2010) further speculated that all close-in giant planets are transported inward by processes that disrupt spin-orbit alignment. Subsequently, the angular momenta are realigned via tidal interaction, and this process is more rapid in cooler stars perhaps due to their thicker convective envelopes. In this picture, any viable migration process would have to introduce misalignment between orbital and stellar spin.

However, the small sample of accurate and precise measurements of stellar obliquities $(\approx 25$ systems $)$ and the possibility of selection effects present us with many pitfalls if we want to validate or reject theories of giant planet migration. Here, we report on our attempts to measure the spin-orbit angles in the WASP-1 and WASP-2 systems, taking advantage of the Rossiter-McLaughlin (RM) effect.

$W A S P-1 b$ was discovered by Cameron et al. (2007). It orbits on a 2.52 circular orbit around an F7V star and has a mass of $0.92 M_{\text {Jup }}$. One reason why this system is interesting is that Stempels et al. (2007) reported a projected stellar rotation speed of $v \sin i_{\star}<5.79 \pm 0.35 \mathrm{~km} \mathrm{~s}^{-1}$, which is relatively slow for a star of this spectral type. For this reason, Schlaufman (2010) identified WASP-1 as a likely case of spin-orbit misalignment along the line of sight, i.e., $\sin i_{\star}<1$ even though $\sin i_{\mathrm{o}} \approx 1$ for the planetary orbit. The star's effective temperature places 


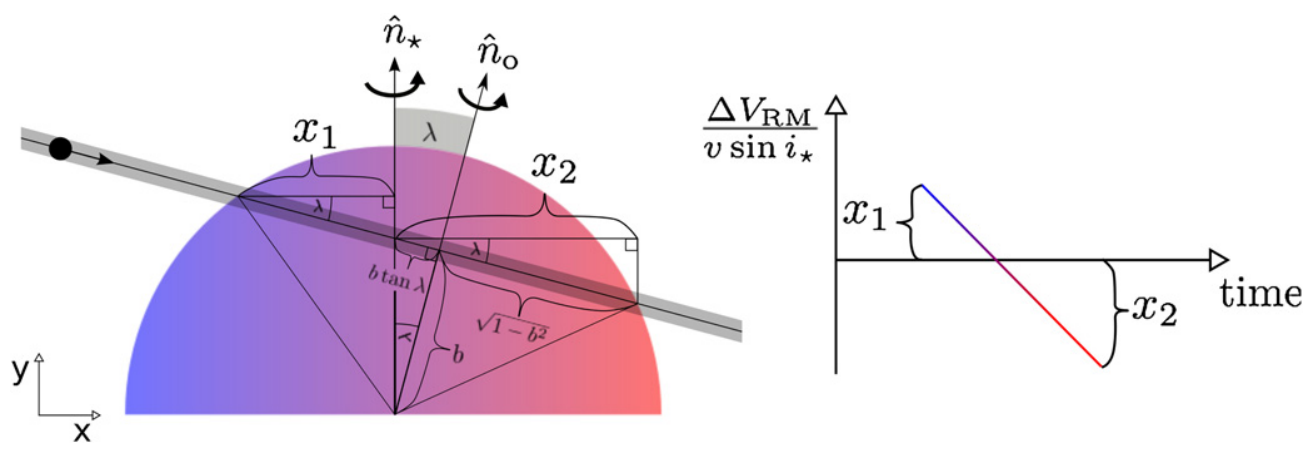

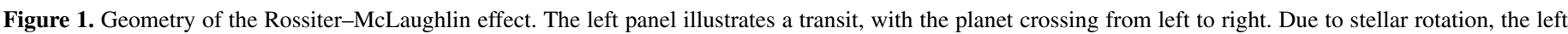

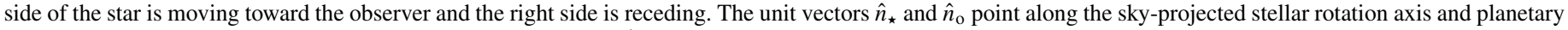

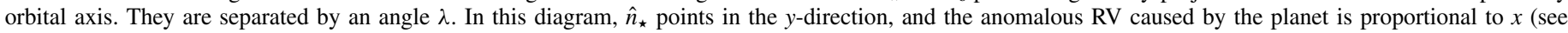

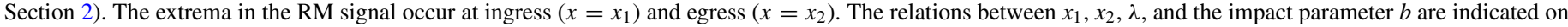
the diagram. The right panel shows the corresponding RM signal as a function of time for an idealized case with no stellar limb darkening.

(A color version of this figure is available in the online journal.)

it right in the range where the transition from well aligned to misaligned orbits was observed by Winn et al. (2010) and Schlaufman (2010). Recently, Simpson et al. (2011) reported a detection of the RM effect for this system and concluded the orbital and stellar spins were misaligned in the plane of the sky. As we will discuss in Section 3, our analysis leads to a more complex conclusion: while we agree that the spin and orbital vectors are misaligned, the evidence for a sky-plane misalignment is much weaker than the evidence for a line-ofsight misalignment.

WASP- $2 b$ was also discovered by Cameron et al. (2007). This $0.87 M_{\text {Jup }}$ planet has a host star of later spectral type (K1V) and orbits on a circular 2.15 orbit. Recently, Triaud et al. (2010) reported an angle of $153_{-15}^{+11}$ deg between the projected orbital and stellar spins, i.e., a retrograde orbit. This is interesting as the host star is firmly on the "cool" side of the proposed divide between cool well aligned stars and hot misaligned stars. WASP2 would therefore constitute an important exception to the trend. However, as we will discuss in Section 4, we find no evidence for a retrograde orbit and argue that the obliquity of the host star cannot be determined from either the new data or the previously published data.

\section{ROSSITER-MCLAUGHLIN EFFECT}

From the perspective of this study, there are two main differences between the WASP-1 and WASP-2 systems. First, the stars are of differing spectral type, leading to different a priori expectations for the stellar rotation speed. The implications of this difference are discussed in Sections 3 and 4. Second, the planets' trajectories across the stellar disk have different impact parameters: WASP- $1 \mathrm{~b}$ nearly crosses the center of the disk, while the transit of WASP-2b is off-center. This section is concerned with the implications of this geometrical difference, as well as the more general relation between the characteristics of the RM signal and the parameters that are often used to model the signal. Some of these aspects of RM modeling were described by Gaudi \& Winn (2007), to which we refer the reader for a more comprehensive account.

Models of the RM effect with varying degrees of accuracy have been worked out by Hosokawa (1953), Queloz et al. (2000), Ohta et al. (2005), Winn et al. (2005), Giménez (2006), Albrecht et al. (2007), Gaudi \& Winn (2007), Collier Cameron et al. (2010), Hirano et al. (2010), and Shporer \& Brown (2011). Because our aim in this section is pedagogical, we ignore the influence of stellar limb-darkening, differential rotation, gravity darkening, surface velocity fields, and any departures from sphericity of the planet or star. We also assume that the planet-to-star radius ratio $R_{p} / R_{\star}$ is small, and that this parameter is known precisely along with all the other parameters that are derived from photometric observations of transits. In particular, we assume precise knowledge of the impact parameter $b \equiv r_{t} \cos i_{\mathrm{o}} / R_{\star}$, where $r_{t}$ is the orbital distance at the time of transit, $R_{\star}$ is the stellar radius, and $i_{\mathrm{o}}$ is the orbital inclination.

With these approximations, the anomalous radial velocity (RV) due to the RM effect is

$$
\Delta V_{\mathrm{RM}}(t) \approx-\left(\frac{R_{p}}{R_{\star}}\right)^{2} v_{p}(t),
$$

where $v_{p}(t)$ is the "subplanet" RV, i.e., the radial component of the rotational velocity of the portion of the photosphere hidden by the planet. Neglecting differential rotation, we may write

$$
v_{p}(t)=\left(v \sin i_{\star}\right) x / R_{\star},
$$

where $x$ is the distance on the sky plane from the center of the planet to the stellar rotation axis (see, e.g., p. 461-462 of Gray 2005).

The situation is illustrated in Figure 1. In this diagram, $\hat{n}_{\star}$ and $\hat{n}_{\mathrm{o}}$ are unit vectors parallel to the sky projections of the stellar and orbital angular momenta, respectively. The angle $\lambda$ is measured from $\hat{n}_{\star}$ to $\hat{n}_{\mathrm{o}} .{ }^{11}$ The maximum redshift and blueshift occur at ingress and egress, which we take to have $x$-coordinates of $x_{1}$ and $x_{2}$, respectively. Using the geometrical relations shown in the diagram, we may write $x_{1}$ and $x_{2}$ in terms of $b$ and $\lambda$ :

$$
\begin{aligned}
& x_{1}=\left(\sqrt{1-b^{2}}-b \tan \lambda\right) \cos \lambda=\sqrt{1-b^{2}} \cos \lambda-b \sin \lambda, \\
& x_{2}=\left(\sqrt{1-b^{2}}+b \tan \lambda\right) \cos \lambda=\sqrt{1-b^{2}} \cos \lambda+b \sin \lambda .
\end{aligned}
$$

It is instructive to examine the (scaled) sum and difference of $x_{1}$ and $x_{2}$ :

$$
\begin{aligned}
& \frac{1}{2} v \sin i_{\star}\left(x_{2}+x_{1}\right)=\sqrt{1-b^{2}} v \sin i_{\star} \cos \lambda, \\
& \frac{1}{2} v \sin i_{\star}\left(x_{2}-x_{1}\right)=b v \sin i_{\star} \sin \lambda .
\end{aligned}
$$

\footnotetext{
11 This definition of $\lambda$ is taken from Ohta et al. (2005). Some other investigators measure the angle from $\hat{n}_{\mathrm{o}}$ to $\hat{n}_{\star}$ and denote the angle $\beta$. Clearly, $\beta=-\lambda$.
} 


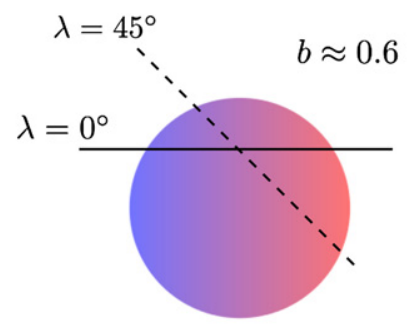

e.g. WASP-2

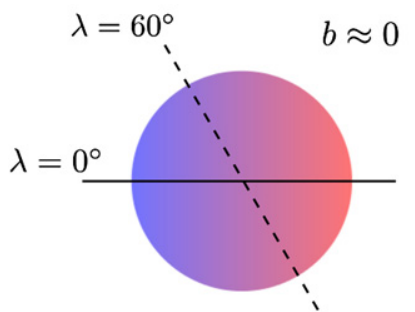

e.g. WASP-1

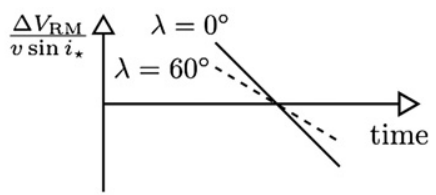

Figure 2. Dependence of the RM signal on $\lambda$ for high and low impact parameters. The upper left panel shows the geometry for a system with $b \approx 0.6$ for two different cases of $\lambda$. In the first case (solid line), the orbital and stellar spins are aligned, and in the second case (dashed line) they are misaligned. The upper right panel shows the corresponding RM signals; both the mean amplitude and the asymmetry of the RM signal are different. The two lower panels show a similar orbital configurations but for $b \approx 0$. Here, the mean amplitude changes with $\lambda$ but the asymmetry is always zero.

(A color version of this figure is available in the online journal.)

The sum is the mean amplitude of the red and blue peaks of the RM effect, while the difference is a measure of asymmetry between the peaks. For a fixed $b$, the mean amplitude depends on $v \sin i_{\star} \cos \lambda$ while the asymmetry depends on $v \sin i_{\star} \sin \lambda$.

Figure 2 shows the RM signal in four different situations: two different values of $\lambda$ for each of two different impact parameters. The upper panels show the case $b \approx 0.6$, as is the case for WASP-2. Here, as $\lambda$ is varied, both the mean amplitude and the asymmetry of the RM signal are observed to change. By measuring the mean amplitude and asymmetry, one may determine both $v \sin i_{\star}$ and $\lambda$. The lower panels show the case $b \approx 0$, as is the case for WASP-1. Here, the asymmetry vanishes regardless of $\lambda$. The only observable quantity is the mean amplitude, and therefore the only parameter combination that can be determined is $v \sin i_{\star} \cos \lambda$.

Consequently, for transits with low impact parameters, $\lambda$ and $v \sin i_{\star}$ have strongly correlated uncertainties and it is not possible to measure $\lambda$ without some prior information about $v \sin i_{\star}$. However, in such cases it is still possible to tell whether $\cos \lambda$ is positive or negative, and therefore whether the orbit is prograde $\left(|\lambda|<90^{\circ}\right)$ or retrograde $\left(|\lambda|>90^{\circ}\right)$. We also note that the degeneracy between $v \sin i_{\star}$ and $\lambda$ can be broken in principle when the RM effect is modeled at the level of spectralline distortion, rather than modeling only the anomalous RV (Albrecht et al. 2007; Collier Cameron et al. 2010). In this paper, though, we work with the anomalous RV.

\section{WASP-1}

\subsection{Observations and Basic Stellar Parameters}

We conducted spectroscopic observations of WASP-1 transits with the Keck I $10 \mathrm{~m}$ telescope and the Subaru $8.2 \mathrm{~m}$ telescope. With Keck, we used the High Resolution Spectrograph (HIRES; Vogt et al. 1994) to gather 34 spectra spanning the transit of 2007 September 1/2. With Subaru, we used the High Dispersion Spectrograph (HDS; Noguchi et al. 2002) to observe two

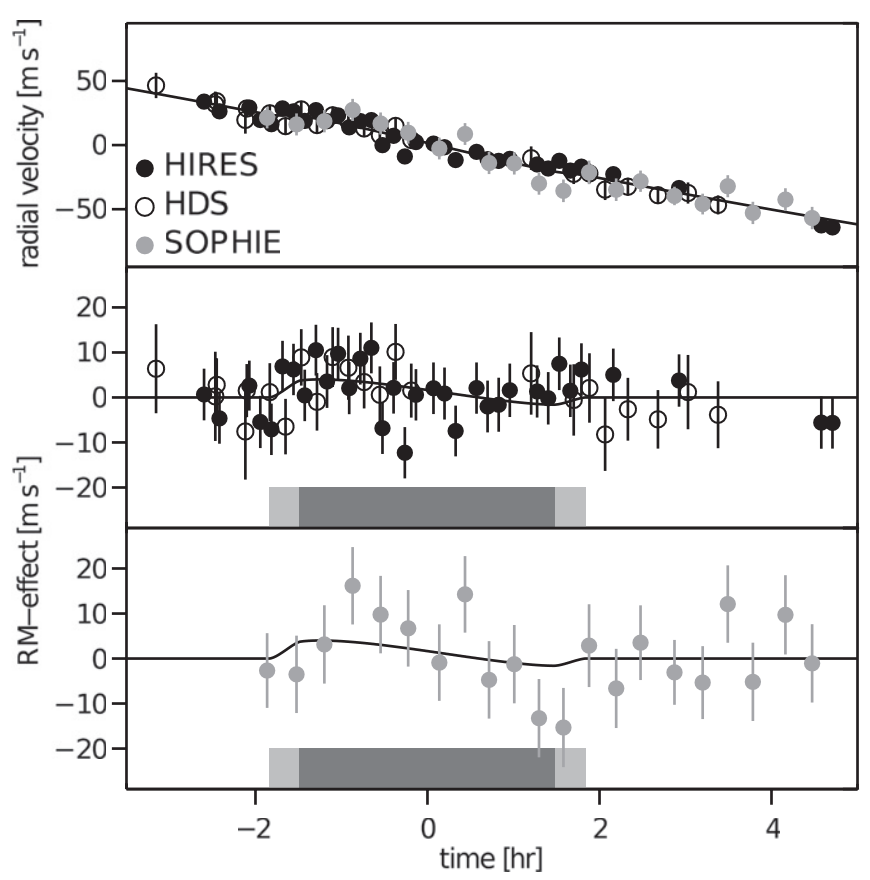

Figure 3. Spectroscopy of WASP-1 transits. The RVs measured before, during, and after transit are plotted as a function of time from inferior conjunction. Solid symbols are data from HIRES and open symbols are data from HDS. Gray symbols are the SOPHIE data from Simpson et al. (2011), which are shown for comparison only (they were not used in our fitting process). The upper panel shows the measured RVs and the best-fitting model. In the middle panel, the orbital contribution to the observed RVs has been subtracted, isolating the RM effect. The lower panel shows the SOPHIE RVs after subtracting our best-fitting orbital model. The light and dark gray bars in the two lower panels indicate times of first, second, third, and fourth contact.

different transits on the nights of 2007 August 4/5 and 2007 September 6/7. A total of 23 spectra were obtained with HDS, most of which (20) were obtained on the latter night. At both observatories, an iodine gas absorption cell was used to correct for changes in the point-spread function and wavelength scale. RVs were derived from the spectra using procedures similar to those described by Butler et al. (1996). See Sato et al. (2002) and Narita et al. (2007) for details on the Subaru data reduction. The RVs are shown in Figure 3 and given in Table 3.

To check on the basic stellar parameters, we used the Spectroscopy Made Easy (SME) software package (Valenti \& Piskunov 1996) to model the high-resolution, high-signal-tonoise ratio template spectrum. We obtained $T_{\text {eff }}=6213 \pm 51 \mathrm{~K}$, $\log g=4.19 \pm 0.07,[\mathrm{M} / \mathrm{H}]=0.17 \pm 0.05$, and $v \sin i_{\star}=$ $1.60 \pm 0.50 \mathrm{~km} \mathrm{~s}^{-1}$. These can be compared to the previous spectroscopic results of WASP-1 by Stempels et al. (2007), which gave $T_{\text {eff }}=6110 \pm 45 \mathrm{~K}, \log g=4.28 \pm 0.15$, $[\mathrm{M} / \mathrm{H}]=0.23 \pm 0.08$, and $v \sin i_{\star}=5.79 \pm 0.35 \mathrm{~km} \mathrm{~s}^{-1}$. Our analysis gave a higher value of $T_{\text {eff }}$ and a lower value of $v \sin i_{\star}$.

The discrepancy in $T_{\text {eff }}$ is discussed in Section 5. The discrepancy in $v \sin i_{\star}$ is of immediate importance because stellar rotation is a key parameter in the interpretation of the RM effect. Frequently, such discrepancies arise because of differing assumptions regarding turbulent broadening. SME determines $v \sin i_{\star}$ based on the observed widths of numerous weak lines in the spectrum. The widths are influenced not only by rotation, but also by random motions of the stellar photosphere (microturbulence and macroturbulence), and these effects cannot generally be disentangled. Hence, it is necessary to assume "typical" values of the turbulence parameters and 


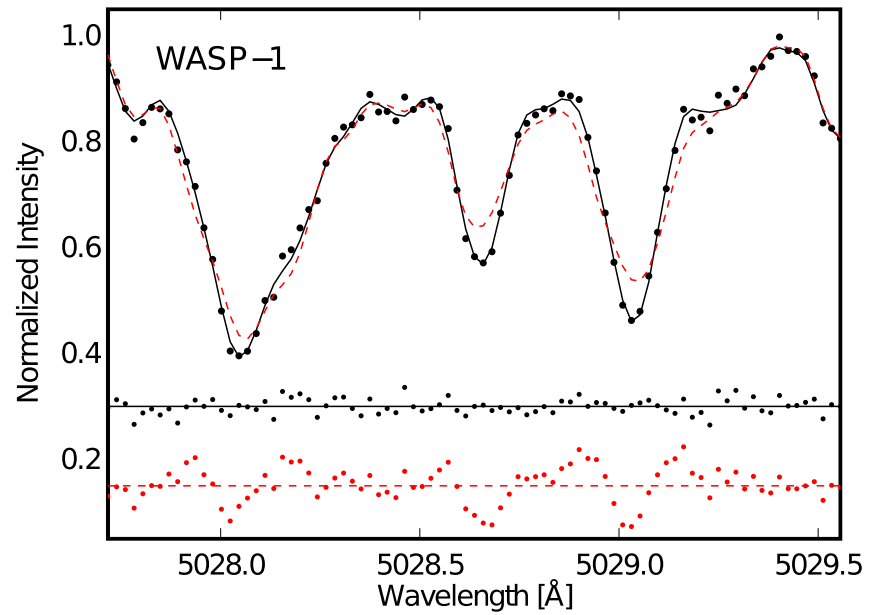

Figure 4. Spectrum of the F7V star WASP-1. A small portion of the spectrum of WASP-1, as obtained with HIRES, is shown. The dots represent the observed spectrum, the solid line represents our best fit with a macroturbulence parameter of $3.98 \mathrm{~km} \mathrm{~s}^{-1}$ and a $v \sin i_{\star}$ of $2.9 \mathrm{~km} \mathrm{~s}^{-1}$. The (red) dashed line shows the spectrum broadened to the values given by Stempels et al. (2007), who obtained a $v \sin i_{\star}$ of $5.79 \mathrm{~km} \mathrm{~s}^{-1}$ with a macroturbulence parameter of $4.5 \mathrm{~km} \mathrm{~s}^{-1}$. The two lower rows of points show the differences between model and data for our best fit (black dots) and the values given by Stempels et al. (2007) (red dots).

(A color version of this figure is available in the online journal.)

attribute the excess broadening of the observed lines to rotation. When using SME, it is assumed $v_{\text {mic }}=0.85 \mathrm{~km} \mathrm{~s}^{-1}$ and

$$
v_{\mathrm{mac}}=\left(3.98+\frac{T_{\mathrm{eff}}-5770 \mathrm{~K}}{650 \mathrm{~K}}\right) \mathrm{km} \mathrm{s}^{-1},
$$

an empirical relation determined by Valenti \& Fischer (2005). ${ }^{12}$ For WASP-1, this formula gives $v_{\mathrm{mac}}=4.66 \mathrm{~km} \mathrm{~s}^{-1}$. This is not too different from the value $v_{\mathrm{mac}}=4.5 \mathrm{~km} \mathrm{~s}^{-1}$ that was assumed by Stempels et al. (2007) and hence the discrepancy in $v \sin i_{\star}$ cannot be attributed to different assumptions regarding macroturbulence.

To investigate further, we performed a differential assay for rotation, based on a comparison between the solar spectrum and a Keck/HIRES spectrum of WASP-1. First, we deconvolved the WASP-1 spectrum to remove the instrumental broadening of width $2.2 \mathrm{~km} \mathrm{~s}^{-1}$. Then, using the MORPH code of Johnson et al. (2006), we applied a rotational broadening kernel to the NSO solar spectrum of Kurucz et al. (1984) to achieve the best fit to the deconvolved WASP-1 spectrum. We found that the best-fitting broadening kernel was $2.36 \mathrm{~km} \mathrm{~s}^{-1}$, indicating the WASP-1 lines are slightly broader than the solar lines. Figure 4 shows a small portion of the WASP-1 spectrum and our bestfitting model based on the broadened solar spectrum.

The larger breadth of the WASP-1 lines could be interpreted as more rapid rotation than the Sun, but in fact part of the increased breadth is expected to be due to the higher macroturbulence of WASP-1. However since the accuracy of Equation (5) is not known, we may here simply assume that the macroturbulence of WASP-1 is greater than or equal to the macroturbulence of the Sun. The MORPH finding implies

$$
\begin{aligned}
& {\left[v \sin i_{\star}(\mathrm{W} 1)\right]^{2}+\left[v_{\mathrm{mac}}(\odot)\right]^{2}} \\
& \quad \approx\left[v \sin i_{\star}(\odot)\right]^{2}+\left[v_{\mathrm{mac}}(\odot)\right]^{2}+\left(2.36 \mathrm{~km} \mathrm{~s}^{-1}\right)^{2}
\end{aligned}
$$

\footnotetext{
12 The equation given here corrects a sign error in Equation (1) of Valenti \&
} Fischer (2005). where the "W1" quantity is for WASP-1 and the " $\odot$ " quantities are for the Sun. ${ }^{13}$ Taking the disk-integrated rotation and macroturbulence of the Sun to be $1.63 \mathrm{~km} \mathrm{~s}^{-1}$ and $3.98 \mathrm{~km} \mathrm{~s}^{-1}$, and the macroturbulence for WASP-1 the same as the Sun, Equation (6) gives $v \sin i_{\star}<2.9 \mathrm{~km} \mathrm{~s}^{-1}$ for WASP-1.

These results show that the projected rotation speed of WASP-1 is quite slow $\left(<2.9 \mathrm{~km} \mathrm{~s}^{-1}\right)$ and is in fact nearly undetectable against the dominant line-broadening effect of macroturbulence. Figure 4 also shows that our spectrum is incompatible with the more rapid rotation of $5.79 \pm 0.35 \mathrm{~km} \mathrm{~s}^{-1}$ found by Stempels et al. (2007). We do not know why Stempels et al. (2007) found a higher $v \sin i_{\star}$ even when making equivalent assumptions regarding macroturbulence. Genuine changes in $v \sin i_{\star}$ could be produced by spin precession, but are not expected to be appreciable on such short timescales, and hence we proceed under the assumption that the Stempels et al. (2007) determination was in error.

To reduce the uncertainties in the photometric parameters, we gathered new photometric data with Keplercam, a CCD camera on the $1.2 \mathrm{~m}$ telescope of the Fred L. Whipple Observatory on Mount Hopkins, Arizona (Szentgyorgyi et al. 2005). Observations were conducted in the Sloan Digital Sky Survey (SDSS) $z^{\prime}$-band on 2009 September 16/17 and 2010 September 29/30, although bad weather interrupted the transit in both cases. The new photometric data were combined with the previous data of Charbonneau et al. (2007), which were gathered with the same instrument and reduced with similar procedures. All of the Keplercam data are shown in Figure 5.

\subsection{Analysis}

To derive constraints on $\lambda$, we fitted a model simultaneously to the RV data and the photometric data. The photometric transit was modeled with the code of Mandel \& Agol (2002), and the RM effect was modeled with a simplified version of the code of Albrecht et al. (2007). This model for the RM effect is similar to that given in Equation (7) but takes limb darkening into account. It does not take into account the nonlinear relation between $\Delta V_{\mathrm{RM}}$ and $v_{p}(t)$ because those nonlinearities are important only for stars with larger $v \sin i_{\star}$ (see, e.g., Winn et al. 2005; Hirano et al. 2010).

The transit impact parameter for WASP-1b is small, with Torres et al. (2008) having reported $b=0.00_{-0.00}^{+0.27}$. Therefore, based on the reasoning of Section 2, we expect the data to constrain $v \sin i_{\star} \cos \lambda$ but not $v \sin i_{\star} \sin \lambda$. For this reason, we chose to parameterize the RM effect with the quantities $\sqrt{v \sin i_{\star}} \cos \lambda$ and $\sqrt{v \sin i_{\star}} \sin \lambda$, rather than $v \sin i_{\star}$ and $\lambda$. The reason for the square roots is to give a constant Jacobian between the fitting parameters and the "physical" parameters $v \sin i_{\star}$ and $\lambda$. As a result, uniform priors in our fitting parameters correspond to the desired uniform priors in $v \sin i_{\star}$ and $\lambda$. With no square roots, and no other adjustment to the fitting procedure, the implicit prior would be linear in $v \sin i_{\star}$ and would thereby bias the results toward faster rotation rates.

The other model parameters were a constant RV offset specific to each spectrograph; the semiamplitude of the star's orbital velocity $\left(K_{\star}\right)$, which controls the RV slope that is observed on each transit night; the orbital period $(P)$; a particular time of midtransit $\left(T_{\mathrm{c}}\right)$; the stellar radius in units of the orbital distance $\left(R_{\star} / a\right)$; the cosine of the orbital inclination $\left(\cos i_{\mathrm{o}}\right)$;

\footnotetext{
13 We verified with numerical experiments that in this regime of velocity widths and for the $\mathrm{S} / \mathrm{N}$ and resolution of our spectrum, the widths of the various convolution kernels can be approximately added in quadrature as implied here.
} 


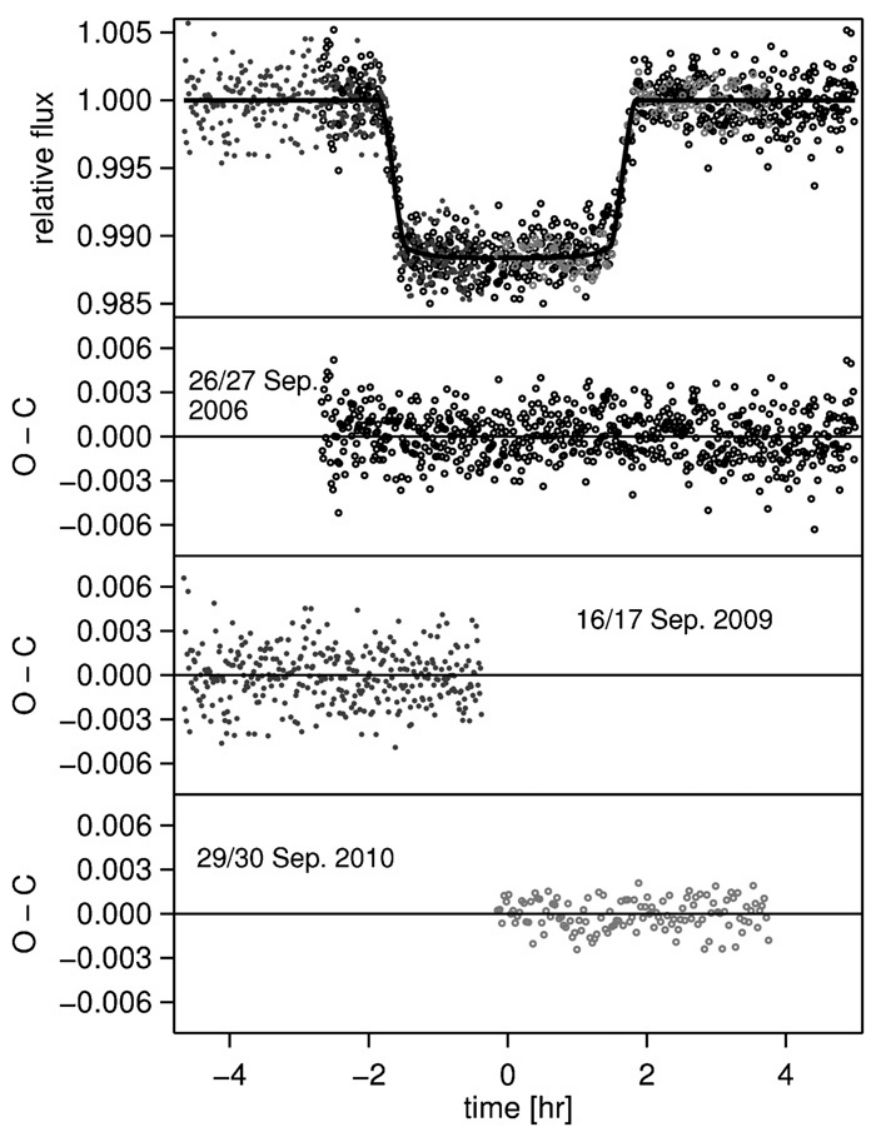

Figure 5. Photometry of WASP-1 transits. The upper panel is a composite $z^{\prime}$-band light curve based on our data and those of Charbonneau et al. (2007). The lower three panels show the residuals between each of the three data sets and the best-fitting model.

the planet-to-star radius ratio $\left(R_{p} / R_{\star}\right)$; two quadratic limbdarkening coefficients $u_{1}$ and $u_{2}$ for describing the $z^{\prime}$-band photometric data; and a linear limb-darkening coefficient $u$ to describe the spectroscopic transit (for which most of the signal is derived from the region 5000-6200 A). According to the tables of Claret (2004), appropriate choices for the limb-darkening coefficients are $u_{1}=0.1666, u_{2}=0.3583$, and $u=0.6$. We allowed $u_{1}+u_{2}$ to be a free parameter and held $u_{1}-u_{2}$ fixed at the tabulated value of -0.1917 , since the difference is only weakly constrained by the data (and in turn has little effect on the other parameters). Likewise we held $u=0.6$ fixed. We assumed the orbit to be circular, as no sign of any eccentricity was detected by Cameron et al. (2007), Madhusudhan \& Winn (2009), Wheatley et al. (2010), or Pont et al. (2011). ${ }^{14}$ All of the time stamps of the spectroscopic and photometric data were placed on the $\mathrm{BJD}_{\mathrm{TDB}}$ system using the algorithm of Eastman et al. (2010).

The fitting statistic was

$$
\begin{aligned}
\chi^{2}= & \sum_{i=1}^{57}\left[\frac{\mathrm{RV}_{i}(o)-\mathrm{RV}_{i}(\mathrm{c})}{\sigma_{\mathrm{RV}, i}}\right]^{2}+\sum_{j=1}^{1134}\left[\frac{F_{j}(o)-F_{j}(c)}{\sigma_{\mathrm{F}, j}}\right]^{2} \\
& +\left(\frac{K_{\star}-115 \mathrm{~m} \mathrm{~s}^{-1}}{11 \mathrm{~m} \mathrm{~s}^{-1}}\right)^{2},
\end{aligned}
$$

\footnotetext{
${ }^{14}$ In particular, Madhusudhan \& Winn (2009) reported an upper limit of $e<0.088$ with $95.4 \%$ confidence. If the orbit were actually eccentric, in contradiction to our modeling assumption, then the main change would be that our result for the velocity semiamplitude $K_{\star}$ would be biased. The results for the spin-orbit parameters would not be significantly affected.
}

Table 1

\begin{tabular}{|c|c|}
\hline Parameter & Values \\
\hline \multicolumn{2}{|c|}{ Parameters mainly derived from photometry } \\
\hline Midtransit time $T_{\mathrm{c}}\left[\mathrm{BJD}_{\mathrm{TDB}}-2,400,000\right]$ & $54461.8630 \pm 0.0002$ \\
\hline Period, $P$ (days) & $2.5199464 \pm 0.0000008$ \\
\hline $\cos i_{\mathrm{o}}$ & $0.000 \pm 0.034$ \\
\hline Fractional stellar radius, $R_{\star} / a$ & $0.173 \pm_{0.001}^{0.003}$ \\
\hline Fractional planetary radius, $R_{p} / R_{\star}$ & $0.1059 \pm 0.0006$ \\
\hline$u_{1}+u_{2}$ & $0.20 \pm 0.05$ \\
\hline \multicolumn{2}{|c|}{ Parameters mainly derived from RVs } \\
\hline Velocity offset, HDS $\left(\mathrm{m} \mathrm{s}^{-1}\right)$ & $0 \pm 1.5$ \\
\hline Velocity offset, HIRES $\left(\mathrm{m} \mathrm{s}^{-1}\right)$ & $-17 \pm 2$ \\
\hline Velocity semiamplitude, $K_{\star}\left(\mathrm{m} \mathrm{s}^{-1}\right)$ & $125 \pm 5$ \\
\hline$\sqrt{v \sin i_{\star}} \sin \lambda\left(\mathrm{km} \mathrm{s}^{-1}\right)$ & $-0.6 \pm 0.9$ \\
\hline$\sqrt{v \sin i_{\star}} \cos \lambda\left(\mathrm{km} \mathrm{s}^{-1}\right)$ & $0.31 \pm 0.25$ \\
\hline \multicolumn{2}{|l|}{ Indirectly derived parameters } \\
\hline Orbital inclination, $i_{\mathrm{O}}\left(^{\circ}\right)$ & $88-92$ \\
\hline Full duration, $T_{14}(\mathrm{hr})$ & $3.684 \pm 0.017$ \\
\hline Ingress or egress duration, $T_{12}$ (minutes) & $21.5 \pm_{0.2}^{0.8}$ \\
\hline Projected stellar rotation speed, $v \sin i_{\star}\left(\mathrm{km} \mathrm{s}^{-1}\right)$ & $0.7 \pm_{0.5}^{1.4}$ \\
\hline Projected spin-orbit angle, $\lambda\left(^{\circ}\right)$ & $-59 \pm \pm_{26}^{99}$ \\
\hline
\end{tabular}

Parameters of the WASP-1 System

where the first two terms are sums of squares over the residuals between the observed $(o)$ and calculated $(c)$ values of the RV and relative flux $(F)$, and the last term represents a prior constraint on $K_{\star}$ based on the results of Cameron et al. (2007). Below we will repeat the analysis including the constrain on $v \sin i_{\star}$ found in Section 3.1.

We solved for the model parameters and their uncertainties using the Markov Chain Monte Carlo (MCMC) algorithm (Tegmark et al. 2004). We used a chain length of $2 \times 10^{6}$ steps and set the size of the steps in each parameter yielding an acceptance rate of about $30 \%$. Before running the chain we increased the uncertainties of the HIRES RVs by adding a "stellar jitter" term of $5 \mathrm{~m} \mathrm{~s}^{-1}$ in quadrature to the internally estimated uncertainties. This choice of jitter term produced a reduced $\chi^{2}$ of unity when that data set was fitted alone. In making this step, we have assumed that the extra RV noise is well described as Gaussian and uncorrelated. This is consistent with the appearance of the residuals shown in Figure 3, although we acknowledge there is no guarantee. Table 3 reports the original, internally estimated uncertainties without any jitter term.

The results for the RM parameters are displayed in Figure 6, and the results for all the parameters are given in Table 1. As anticipated, the weak detection (or nondetection) of the RM effect led to tighter bounds on $v \sin i_{\star} \cos \lambda$ than on $v \sin i_{\star} \sin \lambda$. This is why the contours in Figure 6 reach to large values of $v \sin i_{\star}$ for small values of $\cos \lambda\left(\lambda \approx \pm 90^{\circ}\right)$.

In an attempt to break the degeneracy between $v \sin i_{\star}$ and $\lambda$, we refitted the data with a prior constraint on $v \sin i_{\star}$. Based on the results of Section 3.1, we used a one-sided Gaussian prior, taking the value of unity for $v \sin i_{\star}<2.9 \mathrm{~km} \mathrm{~s}^{-1}$ and falling off as a Gaussian function with $\sigma=0.5 \mathrm{~km} \mathrm{~s}^{-1}$ for higher values. The results from this more constrained MCMC analysis are shown in Figure 6 . The modified bounds on $\lambda$ are $-53 \pm_{29}^{98}$. This analysis disfavors $\lambda \approx \pm 90^{\circ}$ as this would require larger $v \sin i_{\star}$. However, it is not possible to tell definitively whether the positive or negative solution is correct. Within the $95 \%$ confidence contour, all prograde orbits are allowed. 


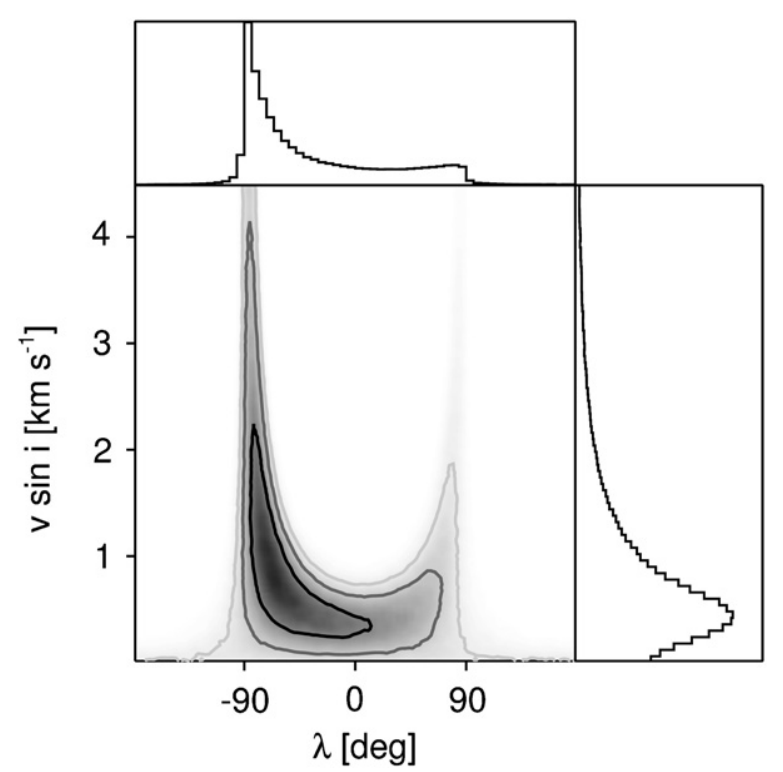

Figure 6. Results for $v \sin i_{\star}$ and $\lambda$, based on our MCMC analysis in the WASP-1 system. The gray scale indicates the posterior probability density, marginalized over all other parameters. The contours represent the twodimensional $68.3 \%, 95 \%$, and $99.73 \%$ confidence limits. The one-dimensional marginalized distributions are shown on the sides of the contour plot. A strong correlation between the projected rotation speed and the projected angle between the stellar and orbital spins exits. Either the two axes are nearly perpendicular on the sky plane, or else $v \sin i_{\star}$ is small and $\lambda$ can have any value.

A different approach is to use a prior constraint on $v$, the actual rotation speed of the star, based on its spectral type and age. Schlaufman (2010) recently presented a formula for a main-sequence star's expected rotation period, given its mass and age. He based the formula on the observed rotation periods of stars in young clusters along with the Skumanich (1972) law $v \propto t^{-1 / 2}$. He further showed that this formula gives a good description of the $v \sin i_{\star}$ distribution of stars in the SPOCS catalog (Valenti \& Fischer 2005). For WASP-1, he found an expected value $v=8.6 \pm 0.5 \mathrm{~km} \mathrm{~s}^{-1}$ where the uncertainty is based only on the uncertainties in the age and mass of WASP-1, and does not account for any uncertainty due to intrinsic scatter in the mass-age-period relation, which seems to be about three times larger than the formal uncertainty (see, e.g., Figure 3 of Schlaufman 2010). Taking $v=8.6 \pm 1.5 \mathrm{~km} \mathrm{~s}^{-1}$ together with our result $v \sin i_{\star}<2.9 \mathrm{~km} \mathrm{~s}^{-1}$, the implication is $\sin i_{\star}<0.34$, i.e., the star is viewed close to pole-on.

One might wonder if the Skumanich law is really applicable to stars with close-in planets, which may have undergone significant rotational evolution due to tidal interactions. For the case of WASP-1, at least, there is supporting evidence for relatively rapid rotation, based on its observed color and chromospheric emission. Aigrain et al. (2004) explain how to use a star's observed $B-V$ and $\log _{10} R_{\mathrm{HK}}^{\prime}$ indices to predict its rotation period. Applied to WASP-1, for which $B-V=0.53$ and $\log _{10} R_{\mathrm{HK}}^{\prime}=-5.114$ (Knutson et al. 2010), we find a rotation period of 12.9 days. Using a stellar radius of $1.45 R_{\odot}$ (Charbonneau et al. 2007), the predicted rotation speed is $v=5.7 \mathrm{~km} \mathrm{~s}^{-1}$, in good agreement with the value expected from the statistical analysis by Schlaufman (2010).

We therefore have two independent lines of evidence for a high obliquity or, equivalently, we have strong evidence against the well aligned scenario in which $\sin i_{\star} \approx 1$ and $\lambda \approx 0^{\circ}$. (1) The absence of a strong RM effect requires either that $|\lambda| \approx$ $90^{\circ}$, or else $v \sin i_{\star}$ is very low $\left(<1 \mathrm{~km} \mathrm{~s}^{-1}\right)$. The latter possibility

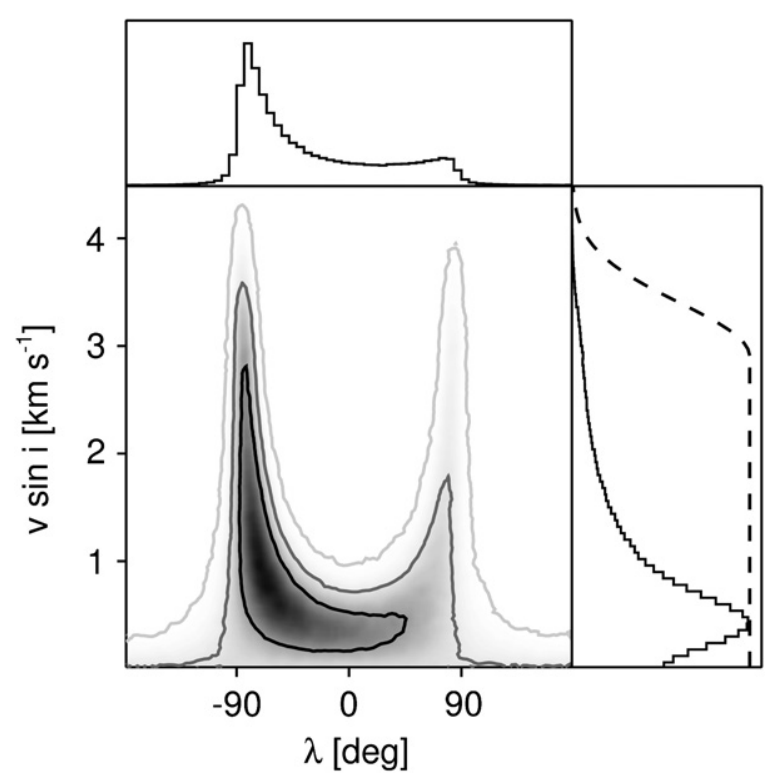

Figure 7. Results for $v \sin i_{\star}$ and $\lambda$, this time including a prior constraint on $v \sin i_{\star}$. The prior constraint was based on the spectroscopic result $v \sin i_{\star}<$ $2.9 \mathrm{~km} \mathrm{~s}^{-1}$ (see Section 3.1) and is illustrated by the dashed line in the righthand side panel. For $v \sin i_{\star}<2.9 \mathrm{~km} \mathrm{~s}^{-1}$, the prior was set equal to unity; and for greater values the prior was a Gaussian function with mean $2.9 \mathrm{~km} \mathrm{~s}^{-1}$ and standard deviation $0.5 \mathrm{~km} \mathrm{~s}^{-1}$. Compared to Figure 6 , the solutions are similar but are constrained to have somewhat lower $v \sin i_{\star}$.

is incompatible with a well aligned $\operatorname{star}\left(\sin i_{\star} \approx 1\right)$, because the rotation rate for a star of the given mass and age is expected to be $8.6 \pm 1.5 \mathrm{~km} \mathrm{~s}^{-1}$. The observed color and chromospheric activity level also suggest a rotation speed of this order. (2) Independently of the RM effect, our determination of $v \sin i_{\star}$ based on the observed width of the spectral lines is much lower than the value of the expected rotation speed, which implies a low $\sin i_{\star}$. In short, it is likely that the stellar and orbital spins are misaligned along the line of sight, and it is possible that they are also misaligned within the sky plane.

\subsection{Comparison with Previous Results}

Simpson et al. (2011) reported $\lambda=-79_{-4.3}^{+4.5}$ for WASP-1b, based on observations taken during and after a planetary transit with the SOPHIE spectrograph on the $1.93 \mathrm{~m}$ telescope of the Observatoire de Haute-Provence. Their value for $\lambda$ is compatible with our result. However, their uncertainty is much smaller than we have found. What causes this difference in obtained confidence intervals?

Their RV data, reproduced in the bottom panel of Figure 3, appear to have a higher amplitude than was seen in our data. This could lead to a somewhat higher result for $v \sin i_{\star}$ but would not by itself affect the very strong correlation between $v \sin i_{\star}$ and $\lambda$. Rather, the important differences are in the methods of analysis. There are two main differences.

First, rather than jointly fitting the photometric and spectroscopic data as we have done, Simpson et al. (2011) fitted their spectroscopic data using independent Gaussian priors on the photometric parameters $a / R_{\star}, R_{p} / R_{\star}$, and $i_{\mathrm{o}}$. The problem is that those parameters are themselves very strongly correlated and their posterior distributions are far from Gaussian. In particular, their photometric priors excluded very low impact parameters, while we find that $b \approx 0$ is allowed. To avoid this problem, it is better to analyze photometric and spectroscopic 


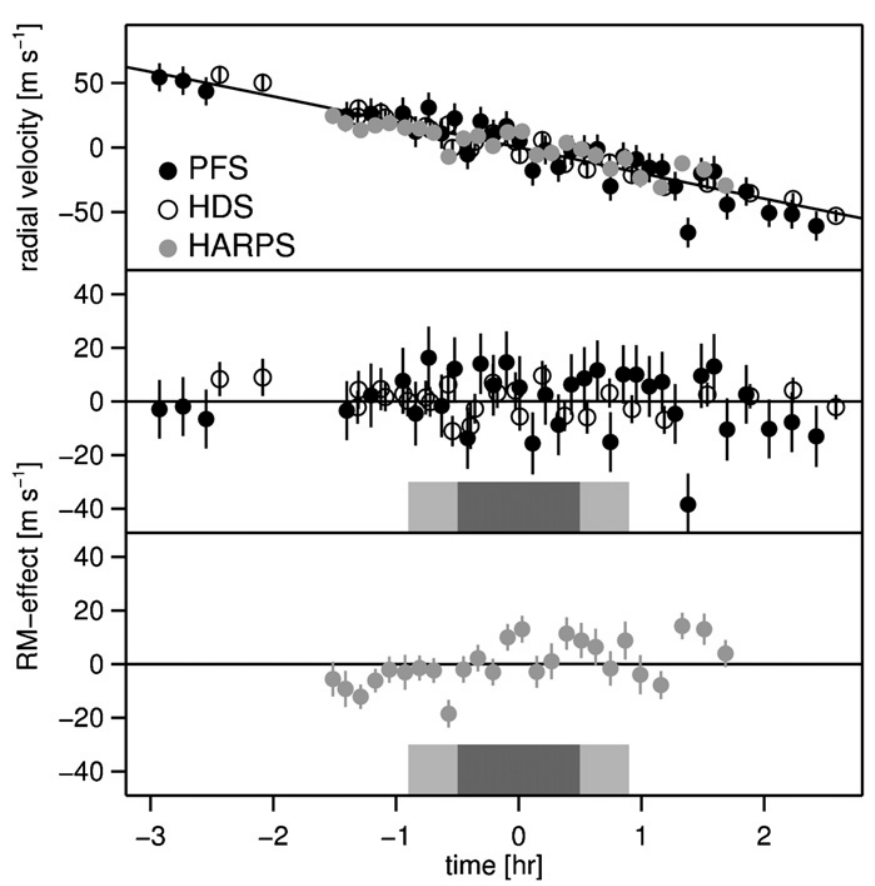

Figure 8. Spectroscopy of WASP-2 transits. Similar to Figure 3. Black symbols are PFS data, and open symbols are HDS data. Gray symbols are the HARPS data of Triaud et al. (2010), which are shown for comparison but were not used during the fitting process. The upper panel shows the data and the best-fitting orbital model. In the lower two panels, our best-fitting orbital model has been subtracted from the data.

data together, or to place priors on the relatively uncorrelated parameters $T_{14}, T_{12}$, and $R_{p} / R_{\star}$ (Carter et al. 2008).

Second, Simpson et al. (2011) used a prior on $v \sin i_{\star}$ based on the spectroscopic analysis of Stempels et al. (2007), which gave $v \sin i_{\star}=5.79 \pm 0.35 \mathrm{~km} \mathrm{~s}^{-1}$. As explained in Section 3.1 and shown in Figure 4, our spectroscopic analysis implies a slower projected rotation rate. Their prior on $v \sin i_{\star}$ pushed their solution toward higher $v \sin i_{\star}$ and excluded aligned configurations of the projected axes.

\section{WASP-2}

\subsection{Observations and Basic Stellar Parameters}

We conducted spectroscopic observations of WASP-2 transits with the Magellan (Clay) $6.5 \mathrm{~m}$ telescope and the Subaru $8.2 \mathrm{~m}$ telescope. With Magellan we used the Planet Finding Spectrograph (PFS; Crane et al. 2010) to gather 35 spectra spanning the transit of 2010 August 26/27. With Subaru we used the HDS to obtain 21 spectra spanning the transit of 2007 September 4/5, and 10 spectra spanning the transit of 2007 September 19/20. Again we employed the iodine-cell technique to derive precise RVs. All the RVs are given in Table 4, and plotted in Figure 8. As was the case for WASP-1, we found no clear evidence for the RM effect.

To check on the basic stellar parameters, we also obtained a high-quality template spectrum with Keck/HIRES, so that we could use the same SME-based analysis that was used for WASP-1. We obtained $T_{\text {eff }}=5206 \pm 50 \mathrm{~K}, \log g=4.51 \pm$ $0.10,[\mathrm{M} / \mathrm{H}]=0.04 \pm 0.05$, and $v \sin i_{\star}=1.3 \pm 0.5 \mathrm{~km} \mathrm{~s}^{-1}$. The assumed macroturbulent velocity was $3.11 \mathrm{~km} \mathrm{~s}^{-1}$. Using the MORPH code described in Section 3.1, we found that the WASP-2 lines are no broader than the solar lines, and estimate $v \sin i_{\star} \lesssim 1.5 \mathrm{~km} \mathrm{~s}^{-1}$.

\subsection{Analysis}

The transit impact parameter for WASP-2b is large, with Torres et al. (2008) having reported $b=0.724_{-0.028}^{+0.017}$. Therefore, based on the reasoning of Section 2, the nondetection of the RM effect implies that both $v \sin i_{\star} \cos \lambda$ and $v \sin i_{\star} \sin \lambda$ are small, which is only possible for low $v \sin i_{\star}$. Unlike the case for WASP-1b, the RM effect for WASP-2 cannot be suppressed by having the planet's trajectory coincide with the sky-projected rotation axis. We therefore expect the nondetection to lead to an upper limit on $v \sin i_{\star}$ and no information about $\lambda$.

For the quantitative analysis, our procedure was similar to that used for WASP-1. The RVs were modeled as the sum of contributions from a circular orbit, the RM effect, and a constant offset specific to each spectrograph. We used a prior on $K_{\star}$ from Triaud et al. (2010), but with a doubled uncertainty (see below), and also tested the sensitivity of the results to this prior as described below. Since the photometric parameters are already precisely determined and we do not have any new photometric data, we implemented priors on the full transit duration $\left(T_{14}\right)$, the ingress or egress duration $\left(T_{12}\right)$, the radius ratio $\left(R_{p} / R_{\star}\right)$ from Charbonneau et al. (2007), and the transit ephemeris based on the analysis of Southworth et al. (2010). The fitting statistic was

$$
\begin{aligned}
\chi^{2}= & \sum_{i=1}^{66}\left[\frac{\mathrm{RV}_{i}(o)-\mathrm{RV}_{i}(c)}{\sigma_{\mathrm{RV}, i}}\right]^{2} \\
& +\left(\frac{T_{\mathrm{c}, \mathrm{BJD}}-2453991.51530}{0.00017}\right)^{2}+\left(\frac{P-2.15222144}{0.00000039}\right)^{2} \\
& +\left(\frac{T_{14}-1.799 \mathrm{hr}}{0.0035 \mathrm{hr}}\right)^{2}+\left(\frac{T_{12}-24.6 \text { minutes }}{2.4 \text { minutes }}\right)^{2} \\
& +\left(\frac{R_{p} / R_{\star}-0.1309}{0.0015}\right)^{2}+\left(\frac{K_{\star}-153.6 \mathrm{~m} \mathrm{~s}^{-1}}{6 \mathrm{~m} \mathrm{~s}^{-1}}\right)^{2}
\end{aligned}
$$

where the symbols have the same meaning as in Section 3. For the PFS data, a "stellar jitter" term of $10 \mathrm{~m} \mathrm{~s}^{-1}$ was added in quadrature to the internally estimated uncertainties to give a reduced $\chi^{2}$ of unity. This probably reflects the limitations of the current algorithm that is used to estimate uncertainties, which is geared toward much brighter stars.

Our results are presented in Table 2 and are illustrated by the contours in the middle panel of Figure 9. (The single solid point in Figure 9 represents the result of Triaud et al. (2010), which will be discussed below.) As expected, $v \sin i_{\star}$ is constrained to low values but $\lambda$ can assume any value from $-180^{\circ}$ to $+180^{\circ}$.

The three different panels of Figure 9 show the results of different choices for the prior on $K_{\star}$. We wondered about the sensitivity of the results to this prior because the star is a latetype star and might be expected to have starspots, which can cause the observed RV slope surrounding the transit phase to be steeper than one would expect from the spectroscopic orbital parameters. Starspots always move across the stellar disk from the approaching limb to the receding limb, and thereby produce an RM-like effect with a negative slope, which is added to the actual orbital velocity gradient. This effect can be seen in a number of RM data sets presented in the literature, most notably for the highly spotted star CoRoT-2 (Bouchy et al. 2008). Depending on the distribution of measurements before, during, and after transit this might introduce different biases in the results for $\lambda$ and $v \sin i_{\star}$.

In Figure 9, the left panel shows the results with no prior on $K_{\star}$, the middle panel shows the result for a prior on $K_{\star}$ as in 

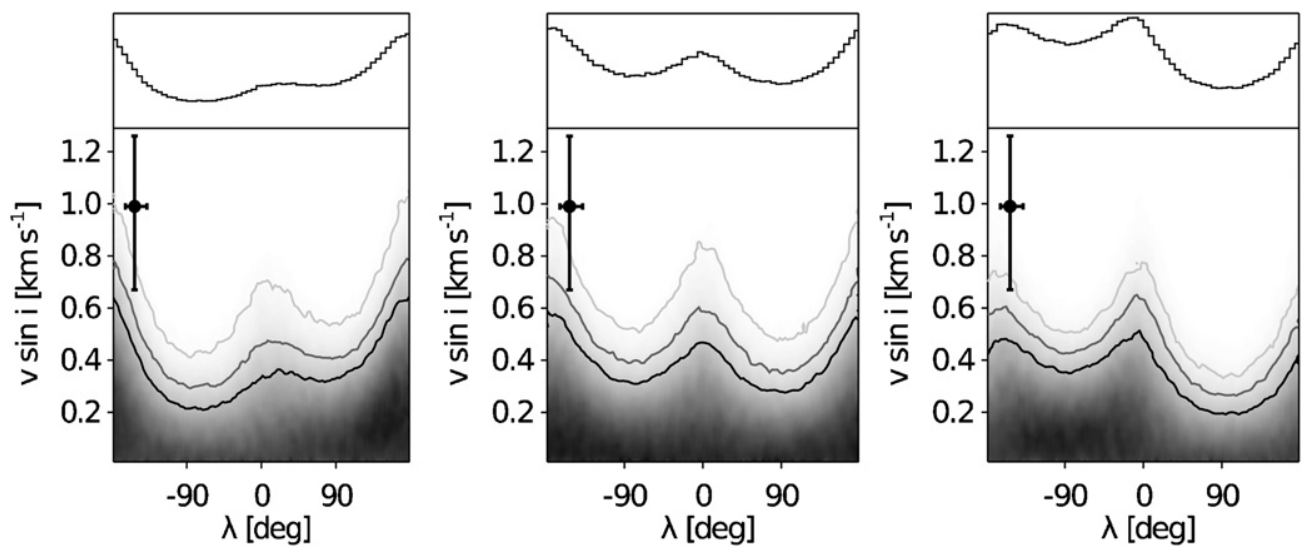

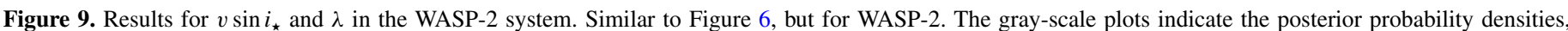

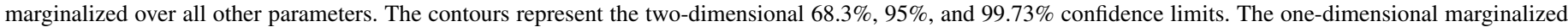

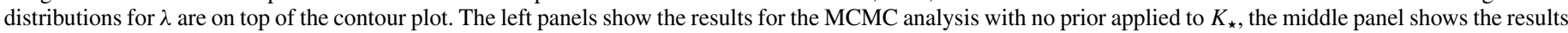

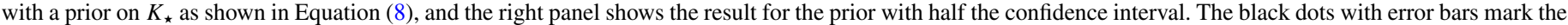

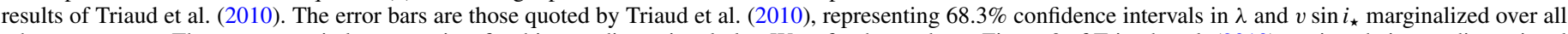

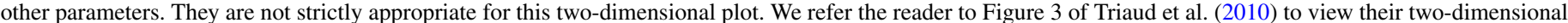
posterior distribution.

Table 2

Parameters of the WASP-2 System

\begin{tabular}{|c|c|}
\hline Parameter & Values \\
\hline \multicolumn{2}{|c|}{ Parameters mainly controlled by prior knowledge } \\
\hline Midtransit time $T_{\mathrm{c}}\left[\mathrm{BJD}_{\mathrm{TDB}}-2,400,000\right]$ & $53991.51530 \pm 0.00017$ \\
\hline Period, $P$ (days) & $2.15222144 \pm 0.00000040$ \\
\hline $\cos i_{\mathrm{o}}$ & $0.091 \pm 0.007$ \\
\hline Fractional stellar radius, $R_{\star} / a$ & $0.125 \pm 0.005$ \\
\hline Fractional planetary radius, $R_{p} / R_{\star}$ & $0.1309 \pm 0.0015$ \\
\hline \multicolumn{2}{|c|}{ Parameters mainly derived from RVs } \\
\hline Velocity offset, PFS $\left(\mathrm{m} \mathrm{s}^{-1}\right)$ & $-2 \pm 2$ \\
\hline Velocity offset, HIRES $\left(\mathrm{m} \mathrm{s}^{-1}\right)$ & $-23.6 \pm 2$ \\
\hline Velocity semiamplitude, $K_{\star}\left(\mathrm{m} \mathrm{s}^{-1}\right)$ & $164 \pm 4$ \\
\hline$\sqrt{v \sin i_{\star}} \sin \lambda\left(\mathrm{km} \mathrm{s}^{-1}\right)$ & $-0.02 \pm 0.28$ \\
\hline$\sqrt{v \sin i_{\star}} \cos \lambda\left(\mathrm{km} \mathrm{s}^{-1}\right)$ & $-0.038 \pm 0.36$ \\
\hline \multicolumn{2}{|c|}{ Indirectly derived parameters } \\
\hline Orbital inclination, $i_{\mathrm{O}}\left({ }^{\circ}\right)$ & $84.8 \pm 0.5$ \\
\hline Full duration, $T_{14}(\mathrm{hr})$ & $1.799 \pm 0.037$ \\
\hline Ingress or egress duration, $T_{12}$ (minutes) & $24.2 \pm 2.4$ \\
\hline Projected stellar rotation speed, $v \sin i_{\star}\left(\mathrm{km} \mathrm{s}^{-1}\right)$ & $<0.5(2 \sigma)$ \\
\hline Projected spin-orbit angle, $\lambda\left({ }^{\circ}\right)$ & All values allowed \\
\hline
\end{tabular}

Equation (8), and the right panel employed the same prior but with a width of $3 \mathrm{~m} \mathrm{~s}^{-1}$ instead of $6 \mathrm{~m} \mathrm{~s}^{-1}$. Evidently, the results are not very sensitive to the prior on $K_{\star}$ : in all cases $v \sin i_{\star}$ must be low and $\lambda$ may have any value. For concreteness, our final results given in Table 2 are based on a prior with a width of $6 \mathrm{~m} \mathrm{~s}^{-1}$ (i.e., the analysis depicted in the middle panel).

One interesting feature of Figure 9 is that the posterior probability density for $\lambda$ has peaks near $0^{\circ}$ and $180^{\circ}$. For these choices of $\lambda$, larger values of $v \sin i_{\star}$ are compatible with the nondetection. This is a general result when fitting RM data with a low signal to noise $(\mathrm{S} / \mathrm{N})$ of a high- $b$ system, and can be understood as follows. For $\lambda$ near $0^{\circ}$ and $180^{\circ}$, the RM signal is antisymmetric about the midtransit time. In such cases $v \sin i_{\star}$ and $K_{\star}$ are strongly correlated parameters, since small changes in either parameter produce changes to the RM signal that are antisymmetric about the midtransit time. This leads to larger confidence intervals for $v \sin i_{\star}$. In contrast, for $\lambda= \pm 90^{\circ}$ the
RM signal is symmetric about the midtransit time; it is a pure redshift or blueshift. Here, the parameters $K_{\star}$ and $v \sin i_{\star}$ are uncorrelated and the allowed region for $v \sin i_{\star}$ shrinks. To put it another way: by fitting for the systemic velocity and $K_{\star}$, we have effectively applied a high-pass filter to the RV data, and thereby reduced the amplitude of any RM signal with $\lambda=90^{\circ}$ in comparison to the higher-frequency signal that is produced with $\lambda$ near $0^{\circ}$ and $180^{\circ}$. This causes the allowed range of $v \sin i_{\star}$ to be higher for $\lambda$ near $0^{\circ}$ and $180^{\circ}$. This explanation was confirmed with further numerical experiments described in Section 4.3.

As with WASP-1, one may try to gain more information on the spin-orbit alignment by using prior constraints on $v \sin i_{\text {太 }}$ or $v$, but in this case not much refinement is possible. The analysis of the WASP-2 template spectrum gives an upper limit $v \sin i_{\star} \lesssim 1.5 \mathrm{~km} \mathrm{~s}^{-1}$ which is not constraining in this context. Also, there have been no reports of photometric variations due to starspots, and hence no stellar rotation period has been determined. Likewise, Schlaufman (2010) found that the expected rotation speed for this system, based on its mass and age, is $1.61 \mathrm{~km} \mathrm{~s}^{-1}$ with an uncertainty range of $1.72 \mathrm{~km} \mathrm{~s}^{-1}$ (presumably an asymmetric error interval). Because of the large uncertainty it is not possible to draw any conclusion about $\sin i_{\star}$, and for this reason Schlaufman (2010) did not identify WASP-2 as a probable case of a misaligned star.

As an additional check on the expected stellar rotation speed, we used the approach of Aigrain et al. (2004) to estimate the rotation period of WASP-2, as we did for WASP-1. In this case, $B-V=0.84$ and $\log _{10} R_{\mathrm{HK}}^{\prime}=-5.054$ (Knutson et al. 2010 ), from which we derive a stellar rotation period of 46 days. Together with a stellar radius of $0.81 R_{\odot}$ (Charbonneau et al. 2007), this gives a rotation speed of $v=0.9 \mathrm{~km} \mathrm{~s}^{-1}$ which is in line with the low speed predicted by Schlaufman (2010).

\subsection{Comparison with Previous Results}

A transit of WASP-2 was observed by Triaud et al. (2010) with the HARPS spectrograph. Their data are shown in the bottom panel of Figure 8. Based on the HARPS data they found $\lambda=-153_{-11}^{+15}$ deg (a retrograde orbit) and $v \sin i_{\star}=$ $0.99_{-0.32}^{+0.27} \mathrm{~km} \mathrm{~s}^{-1}$. Our data are not compatible with those parameters. When we fixed $\lambda$ and $v \sin i_{\star}$ at the values found by these researchers, and refitted our data, the minimum $\chi^{2}$ rose 


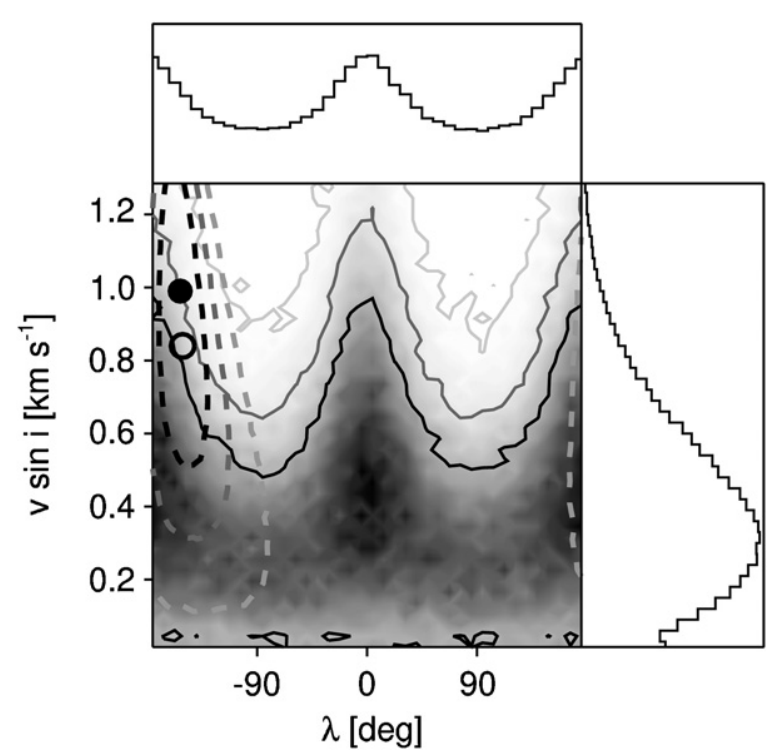

Figure 10. Results for simulated data sets with no RM effect. Similar to Figure 9 , but this time based on the analysis of $2 \times 10^{5}$ simulated data sets with no RM effect but with the same time sampling and roughly the same RV precision as the HARPS data. The gray shades show the density of the best-fitting values of $v \sin i_{\star}$ and $\lambda$. The contours enclose $68.3 \%, 95 \%$, and $99.73 \%$ of the best-fitting values. The solid circle shows the Triaud et al. (2010) result. The open circle and the dashed contours show our results of fitting the actual HARPS data set with our MCMC routine.

from 60.9 to 72.6 , giving $\Delta \chi^{2}=11.7$. What can have caused the difference between our results and theirs?

Daemgen et al. (2009) found that WASP-2 has a neighboring star (a companion or chance alignment) at an angular separation of 0.7 arcsec, close enough to have been possibly included within the spectrograph slit or fiber in some cases. It is hard to predict the exact effect that the additional starlight would have on the spectroscopic analysis, but as the neighbor is 4 mag fainter than WASP-2, and as its spectral type and systemic velocity are likely quite different from that of WASP-2, we consider it unlikely that variable contamination by this star is responsible for the differing results. We are therefore led to look elsewhere for an explanation.

One relevant difference in the analysis procedures is that Triaud et al. (2010) used uniform priors in $v \sin i_{\star} \sin \lambda$ and $v \sin i_{\star} \cos \lambda$, thereby adopting a prior that is linear in $v \sin i_{\star}$. This is in contrast to our prior which was uniform in $v \sin i_{\star}$. Their prior pushes $v \sin i_{\star}$ to higher values and therefore pushes $\lambda$ near $0^{\circ}$ or $180^{\circ}$ (see Figure 9). When we refitted their data using our procedure, we found a lower $v \sin i_{\star}$ and an enlarged confidence interval, as expected. The open circle and the thick dashed lines in Figure 10 represent our fit to the HARPS data. However, this difference in priors cannot explain the entire discrepancy: even our reanalysis of the HARPS data gives $\lambda=-151_{-13}^{+20}$ deg and $v \sin i_{\star}=0.84 \pm 0.35 \mathrm{~km} \mathrm{~s}^{-1}$.

This apparently statistically significant result is surprising since the RM effect is not apparent by visual inspection of the data (Figure 8). The data during the transit do not appear too different from the data outside of the transit. If the RM effect had been measured but not modeled, then one would expect the residuals between the data and the best-fitting orbital model would have a higher scatter inside the transit than outside the transit. For our data, this is not the case. For the HARPS data set, the rms residual of the out-of-transit data is $6.9 \mathrm{~m} \mathrm{~s}^{-1}$, as compared to $7.2 \mathrm{~m} \mathrm{~s}^{-1}$ during transit. This represents only a marginal increase in scatter.
Table 3

Relative Radial Velocity Measurements of WASP-1

\begin{tabular}{|c|c|c|c|}
\hline Time $\left[\mathrm{BJD}_{\mathrm{TDB}}\right]$ & $\mathrm{RV}\left(\mathrm{m} \mathrm{s}^{-1}\right)$ & Unc. $\left(\mathrm{m} \mathrm{s}^{-1}\right)$ & Spectrograph \\
\hline 2454345.83725 & 33.86 & 2.82 & HIRES \\
\hline 2454345.84471 & 26.32 & 2.57 & HIRES \\
\hline 2454345.85916 & 29.23 & 2.40 & HIRES \\
\hline 2454345.86449 & 19.53 & 2.94 & HIRES \\
\hline 2454345.86988 & 16.26 & 2.76 & HIRES \\
\hline 2454345.87525 & 28.59 & 2.71 & HIRES \\
\hline 2454345.88059 & 26.28 & 2.75 & HIRES \\
\hline 2454345.88603 & 18.84 & 2.76 & HIRES \\
\hline 2454345.89146 & 27.23 & 2.63 & HIRES \\
\hline 2454345.89684 & 18.61 & 3.05 & HIRES \\
\hline 2454345.90219 & 23.13 & 2.87 & HIRES \\
\hline 2454345.90758 & 13.82 & 2.92 & HIRES \\
\hline 2454345.91296 & 18.70 & 2.84 & HIRES \\
\hline 2454345.91830 & 19.45 & 2.65 & HIRES \\
\hline 2454345.92368 & -0.07 & 2.78 & HIRES \\
\hline 2454345.92907 & 7.22 & 2.77 & HIRES \\
\hline 2454345.93453 & -8.89 & 2.73 & HIRES \\
\hline 2454345.93990 & 2.32 & 2.74 & HIRES \\
\hline 2454345.94841 & 1.12 & 2.81 & HIRES \\
\hline 2454345.95376 & -1.71 & 2.91 & HIRES \\
\hline 2454345.95912 & -11.69 & 2.60 & HIRES \\
\hline 2454345.96926 & -5.30 & 2.73 & HIRES \\
\hline 2454345.97461 & -11.05 & 2.85 & HIRES \\
\hline 2454345.97998 & -12.33 & 3.32 & HIRES \\
\hline 2454345.98537 & -10.78 & 3.19 & HIRES \\
\hline 2454345.99861 & -15.13 & 2.93 & HIRES \\
\hline 2454346.00397 & -18.29 & 2.98 & HIRES \\
\hline 2454346.00936 & -12.28 & 3.03 & HIRES \\
\hline 2454346.01468 & -19.91 & 2.99 & HIRES \\
\hline 2454346.02006 & -16.84 & 2.99 & HIRES \\
\hline 2454346.03551 & -22.71 & 3.01 & HIRES \\
\hline 2454346.06736 & -33.51 & 2.97 & HIRES \\
\hline 2454346.13628 & -62.64 & 2.96 & HIRES \\
\hline 2454346.14171 & -64.20 & 2.78 & HIRES \\
\hline 2454318.09458 & 63.61 & 9.84 & HDS \\
\hline 2454318.12329 & 48.93 & 9.86 & HDS \\
\hline 2454318.13785 & 36.74 & 10.64 & HDS \\
\hline 2454350.88327 & 51.29 & 5.81 & HDS \\
\hline 2454350.89783 & 45.61 & 5.81 & HDS \\
\hline 2454350.90899 & 41.88 & 6.36 & HDS \\
\hline 2454350.91661 & 31.89 & 6.08 & HDS \\
\hline 2454350.92423 & 44.93 & 6.19 & HDS \\
\hline 2454350.93185 & 32.72 & 6.31 & HDS \\
\hline 2454350.93945 & 40.32 & 6.57 & HDS \\
\hline 2454350.94707 & 35.60 & 7.06 & HDS \\
\hline 2454350.95469 & 30.06 & 5.77 & HDS \\
\hline 2454350.96231 & 24.87 & 6.22 & HDS \\
\hline 2454350.96992 & 32.04 & 6.13 & HDS \\
\hline 2454350.97753 & 21.08 & 5.92 & HDS \\
\hline 2454351.03561 & 6.90 & 9.11 & HDS \\
\hline 2454351.05624 & -5.33 & 7.87 & HDS \\
\hline 2454351.06385 & -5.00 & 7.65 & HDS \\
\hline 2454351.07146 & -17.63 & 8.04 & HDS \\
\hline 2454351.08247 & -15.36 & 6.90 & HDS \\
\hline 2454351.09703 & -21.98 & 6.42 & HDS \\
\hline 2454351.11159 & -20.25 & 8.19 & HDS \\
\hline 2454351.12615 & -29.56 & 7.36 & HDS \\
\hline
\end{tabular}

This led us to conduct some numerical experiments on fitting random noise with similar characteristics to the HARPS data. We used the time stamps of the HARPS transit-night data, and simulated RV data based on only the best-fitting orbital model for WASP-2. We added Gaussian "measurement" uncertainties with a standard deviation of $7.0 \mathrm{~m} \mathrm{~s}^{-1}$. Then we fitted this 
Table 4

Relative Radial Velocity Measurements of WASP-2

\begin{tabular}{|c|c|c|c|}
\hline Time $\left[\mathrm{BJD}_{\mathrm{TDB}}\right]$ & $\mathrm{RV}\left(\mathrm{m} \mathrm{s}^{-1}\right)$ & Unc. $\left(\mathrm{m} \mathrm{s}^{-1}\right)$ & Spectrograph \\
\hline 2454348.72936 & 47.57 & 6.15 & HDS \\
\hline 2454348.73875 & 46.93 & 5.29 & HDS \\
\hline 2454348.74635 & 41.88 & 5.75 & HDS \\
\hline 2454348.75397 & 37.82 & 5.51 & HDS \\
\hline 2454348.76158 & 23.31 & 5.67 & HDS \\
\hline 2454348.76920 & 28.01 & 5.53 & HDS \\
\hline 2454348.77680 & 30.28 & 5.66 & HDS \\
\hline 2454348.78442 & 17.81 & 5.26 & HDS \\
\hline 2454348.79204 & 29.38 & 5.52 & HDS \\
\hline 2454348.79965 & 10.77 & 5.73 & HDS \\
\hline 2454348.80728 & 6.57 & 6.10 & HDS \\
\hline 2454348.81489 & 11.95 & 5.42 & HDS \\
\hline 2454348.82250 & 2.32 & 5.23 & HDS \\
\hline 2454348.83352 & -6.93 & 5.20 & HDS \\
\hline 2454348.84809 & -4.37 & 4.45 & HDS \\
\hline 2454348.86264 & -11.82 & 4.52 & HDS \\
\hline 2454348.87720 & -16.39 & 4.75 & HDS \\
\hline 2454348.89175 & -29.35 & 4.59 & HDS \\
\hline 2454348.90632 & -33.89 & 4.52 & HDS \\
\hline 2454348.92089 & -44.93 & 4.18 & HDS \\
\hline 2454348.95000 & -54.24 & 4.86 & HDS \\
\hline 2454363.74812 & 79.94 & 6.36 & HDS \\
\hline 2454363.76268 & 73.84 & 6.94 & HDS \\
\hline 2454363.79521 & 53.93 & 7.07 & HDS \\
\hline 2454363.80283 & 50.60 & 7.88 & HDS \\
\hline 2454363.81044 & 45.11 & 7.11 & HDS \\
\hline 2454363.81804 & 40.19 & 6.31 & HDS \\
\hline 2454363.82566 & 41.40 & 7.46 & HDS \\
\hline 2454363.83326 & 22.31 & 8.60 & HDS \\
\hline 2454363.84088 & 34.76 & 7.03 & HDS \\
\hline 2454363.84850 & 28.17 & 6.82 & HDS \\
\hline 2455435.53391 & 56.65 & 4.38 & PFS \\
\hline 2455435.54192 & 54.05 & 4.56 & PFS \\
\hline 2455435.54978 & 45.81 & 4.58 & PFS \\
\hline 2455435.59753 & 26.70 & 4.62 & PFS \\
\hline 2455435.60576 & 28.47 & 6.46 & PFS \\
\hline 2455435.61657 & 28.78 & 7.18 & PFS \\
\hline 2455435.62090 & 14.48 & 6.44 & PFS \\
\hline 2455435.62533 & 33.21 & 6.00 & PFS \\
\hline 2455435.62971 & 13.14 & 6.00 & PFS \\
\hline 2455435.63414 & 24.86 & 6.09 & PFS \\
\hline 2455435.63854 & -3.11 & 5.62 & PFS \\
\hline 2455435.64295 & 22.52 & 5.21 & PFS \\
\hline 2455435.64731 & 12.40 & 5.33 & PFS \\
\hline 2455435.65172 & 18.86 & 5.71 & PFS \\
\hline 2455435.65618 & 7.36 & 5.93 & PFS \\
\hline 2455435.66062 & -15.67 & 5.78 & PFS \\
\hline 2455435.66496 & 0.39 & 5.01 & PFS \\
\hline 2455435.66941 & -12.93 & 5.32 & PFS \\
\hline 2455435.67376 & 0.00 & 5.24 & PFS \\
\hline 2455435.67817 & 0.19 & 5.95 & PFS \\
\hline 2455435.68261 & 1.08 & 4.92 & PFS \\
\hline 2455435.68702 & -27.79 & 4.90 & PFS \\
\hline 2455435.69140 & -4.66 & 4.44 & PFS \\
\hline 2455435.69580 & -6.84 & 4.64 & PFS \\
\hline 2455435.70025 & -13.39 & 5.39 & PFS \\
\hline 2455435.70460 & -13.75 & 5.15 & PFS \\
\hline 2455435.70901 & -27.76 & 4.88 & PFS \\
\hline 2455435.71338 & -63.64 & 5.72 & PFS \\
\hline 2455435.71787 & -17.76 & 6.67 & PFS \\
\hline 2455435.72222 & -16.32 & 6.81 & PFS \\
\hline 2455435.72666 & -41.92 & 5.87 & PFS \\
\hline 2455435.73313 & -31.84 & 4.42 & PFS \\
\hline 2455435.74091 & -48.37 & 4.57 & PFS \\
\hline 2455435.74864 & -49.44 & 4.97 & PFS \\
\hline 2455435.75692 & -58.54 & 5.51 & PFS \\
\hline
\end{tabular}

mock data set together with the photometric priors using a Levenberg-Marquardt least-squares minimization routine. This was repeated $2 \times 10^{5}$ times with different realizations of the measurement errors. ${ }^{15}$ The density distribution of the $2 \times 10^{5}$ best-fitting solutions is shown in Figure 10. As discussed in Section 4.2, we found that even though the mock data had no RM effect at all, there is a clear tendency to "find" solutions near $\lambda=0^{\circ}$ or $180^{\circ}$. This should raise a concern about the claimed detection of the RM effect with $\lambda \approx 0^{\circ}$ or $\approx 180^{\circ}$ with a low $\mathrm{S} / \mathrm{N}$. The result of our fitting code applied to the actual HARPS data (open circle and dashed contours in Figure 10) gives values for $v \sin i_{\star}$ and $\lambda$ that are within the area containing $95 \%$ of the mock data solutions. In this sense, the "false alarm" probability (the odds of finding such an apparently significant retrograde orbit when fitting only random noise) is at least $5 \%$. It is probably higher, when one considers that the true noise may not be uncorrelated and Gaussian. We therefore conclude that the current data do not provide secure information on the orientation of the stellar spin relative to the orbital spin.

\section{DISCUSSION AND SUMMARY}

We have presented two nondetections of the RM effect for the transiting planets WASP- $1 \mathrm{~b}$ and WASP- $2 \mathrm{~b}$. In both cases, we gathered high-resolution, high $\mathrm{S} / \mathrm{N}$ spectra on nights spanning transits, using multiple large telescopes. For WASP-1, there is a weak indication of a prograde RM effect, and for WASP-2 we did not detect the RM effect. Due to the differences in the transit geometry, and in the stellar type, we arrived at different conclusions about the relative orientation of the stellar spin and orbit in each case.

Because the transit of WASP-1b has a very low impact parameter, the only way to produce a low-amplitude RM effect is to have nearly perpendicular sky projections of the spin and orbital axes (implying a large misalignment in the sky plane), or to have a very low $v \sin i_{\star}$. The latter option also implies a likely misalignment, because the resulting upper limit on $v \sin i_{\star}$ is lower than the expected $v$ for a star of the given age and mass. A similar comparison can be made between the expected $v$ and the lower $v \sin i_{\star}$ that is estimated from the breadth of spectral absorption lines. Thus, the data give strong evidence for misalignment, although it is not certain whether the misalignment is mainly along the line of sight, or in the sky plane, or both.

For WASP-2b, no information on $\lambda$ was gained from our nondetection, mainly because this star is expected to be a slow rotator. The upper limit on $v \sin i_{\star}$ from the RM nondetection is within the expected range of $v$ for a star of the given mass and age. An analysis of previous HARPS data favored a retrograde orbit for the planet, but we have argued that this may have been a statistical false alarm. Numerical experiments confirm that fitting random noise with an RM model can produce false detections with nearly the same amplitude as the claimed detection. For a firmer conclusion, one would need to gather more spectroscopic data during transits. These same numerical experiments should lead to a re-evaluation of other cases in which the RM effect was detected with low statistical significance, such as TrES-2b (Winn et al. 2008).

We now put these results into the context of the pattern noted by Winn et al. (2010) and Schlaufman (2010) that hot stars tend

\footnotetext{
15 We did not use the MCMC algorithm as it would take too long to make chains for $10^{5}$ data sets, and because we are only interested in the best-fitting values of $v \sin i_{\star}$ and $\lambda$ for each mock data set and not the individual confidence intervals.
} 
to have high obliquity. The proposed boundary line between "hot" and "cool" stars was around $T_{\text {eff }}=6250 \mathrm{~K}$.

For WASP-2, Cameron et al. (2007) measured an effective temperature of $5200 \pm 200 \mathrm{~K}$, and from our HIRES spectrum we found $5206 \pm 50 \mathrm{~K}$. Thus, there is consensus that WASP-2 is a cool star. The finding of a retrograde orbit by Triaud et al. (2010) was a strong exception to the proposed pattern. Our data and our analysis led us to conclude that the spin-orbit angle for this system is undetermined, and therefore that WASP-2 is not an exception.

For WASP-1, Cameron et al. (2007) measured an effective temperature of $6200 \pm 200 \mathrm{~K}$. Further observations and spectroscopic analysis were presented by Stempels et al. (2007), who found $T_{\mathrm{eff}}=6110 \pm 45 \mathrm{~K}$. Our analysis of a HIRES spectrum gave $T_{\text {eff }}=6213 \pm 51 \mathrm{~K}$, or $100 \mathrm{~K}$ hotter than the determination by Stempels et al. (2007). Probably the reason for the difference is that Stempels et al. (2007) used the $\mathrm{H} \alpha$ line profile as the main constraint on $T_{\text {eff }}$, while our analysis used the standard SME wavelength intervals which exclude $\mathrm{H} \alpha$ (Valenti \& Fischer 2005; see their Table 3). It is beyond the scope of this article to evaluate the relative merits of these different methods for establishing an accurate effective temperature scale. Instead we note that the SME-based scale that we have used is similar or identical to the scale that has been used for the other transit-hosting stars, and therefore the scale on which the proposed boundary of $6250 \mathrm{~K}$ is relevant. In this light it seems that WASP-1, with $T_{\text {eff }}(\mathrm{SME})=6213 \pm 51 \mathrm{~K}$, is very near the boundary. Therefore, the finding of a high obliquity neither corroborates nor weakens the proposed pattern, although WASP-1 may serve as a useful point in establishing the sharpness of the transition from mainly misaligned to mainly aligned.

We thank G. Marcy and M. Holman for help gathering some of the data presented here. We are grateful to the anonymous referee for a prompt and insightful report, and to Amaury Triaud for comments on the manuscript. S.A. acknowledges support by a Rubicon fellowship from the Netherlands Organization for Scientific Research (NWO). J.N.W. acknowledges support from a NASA Origins grant (NNX09AD36G). This research has made use of the Simbad database located at http://simbad.u-strasbg.fr/.

Facilities: Keck:I, Subaru, Magellan:Clay

\section{REFERENCES}

Aigrain, S., Favata, F., \& Gilmore, G. 2004, A\&A, 414, 1139

Albrecht, S., Reffert, S., Snellen, I., Quirrenbach, A., \& Mitchell, D. S. 2007, A\&A, 474, 565

Bouchy, F., et al. 2008, A\&A, 482, L25

Butler, R. P., Marcy, G. W., Williams, E., McCarthy, C., Dosanjh, P., \& Vogt, S. S. 1996, PASP, 108, 500
Cameron, A. C., et al. 2007, MNRAS, 375, 951

Carter, J. A., Yee, J. C., Eastman, J., Gaudi, B. S., \& Winn, J. N. 2008, ApJ, 689,499

Charbonneau, D., Winn, J. N., Everett, M. E., Latham, D. W., Holman, M. J., Esquerdo, G. A., \& O’Donovan, F. T. 2007, ApJ, 658, 1322

Claret, A. 2004, A\&A, 428, 1001

Collier Cameron, A., Bruce, V. A., Miller, G. R. M., Triaud, A. H. M. J., \& Queloz, D. 2010, MNRAS, 403, 151

Crane, J. D., Shectman, S. A., Butler, R. P., Thompson, I. B., Birk, C., Jones, P., \& Burley, G. S. 2010, Proc. SPIE, 7735, 170

Daemgen, S., Hormuth, F., Brandner, W., Bergfors, C., Janson, M., Hippler, S., \& Henning, T. 2009, A\&A, 498, 567

Eastman, J., Siverd, R., \& Gaudi, B. S. 2010, PASP, 122, 935

Gaudi, B. S., \& Winn, J. N. 2007, ApJ, 655, 550

Giménez, A. 2006, ApJ, 650, 408

Gray, D. F. 2005, The Observation and Analysis of Stellar Photospheres (3rd ed; Cambridge: Cambridge Univ. Press)

Hébrard, G., et al. 2008, A\&A, 488, 763

Hirano, T., Suto, Y., Taruya, A., Narita, N., Sato, B., Johnson, J. A., \& Winn, J. N. 2010, ApJ, 709, 458

Hosokawa, Y. 1953, PASJ, 5, 88

Johnson, J. A., Winn, J. N., Albrecht, S., Howard, A. W., Marcy, G. W., \& Gazak, J. Z. 2009, PASP, 121, 1104

Johnson, J. A., et al. 2006, ApJ, 647, 600

Knutson, H. A., Howard, A. W., \& Isaacson, H. 2010, ApJ, 720, 1569

Kurucz, R. L., Furenlid, I., Brault, J., \& Testerman, L. 1984, Solar Flux Atlas from 296 to $1300 \mathrm{~nm}$, in National Solar Observatory Atlas (Sunspot, NM: National Solar Observatory)

Lin, D. N. C., Bodenheimer, P., \& Richardson, D. C. 1996, Nature, 380, 606

Madhusudhan, N., \& Winn, J. N. 2009, ApJ, 693, 784

Mandel, K., \& Agol, E. 2002, ApJ, 580, L171

Nagasawa, M., Ida, S., \& Bessho, T. 2008, ApJ, 678, 498

Narita, N., Sato, B., Hirano, T., \& Tamura, M. 2009, PASJ, 61, L35

Narita, N., et al. 2007, PASJ, 59, 763

Noguchi, K., et al. 2002, PASJ, 54, 855

Ohta, Y., Taruya, A., \& Suto, Y. 2005, ApJ, 622, 1118

Pont, F., Husnoo, N., Mazeh, T., \& Fabrycky, D. 2011, MNRAS, 414, 1278

Queloz, D., Eggenberger, A., Mayor, M., Perrier, C., Beuzit, J. L., Naef, D., Sivan, J. P., \& Udry, S. 2000, A\&A, 359, L13

Sato, B., Kambe, E., Takeda, Y., Izumiura, H., \& Ando, H. 2002, PASJ, 54, 873

Schlaufman, K. C. 2010, ApJ, 719, 602

Shporer, A., \& Brown, T. 2011, ApJ, 733, 30

Simpson, E. K., et al. 2011, MNRAS, 414, 3023

Skumanich, A. 1972, ApJ, 171, 565

Southworth, J., et al. 2010, MNRAS, 408, 1680

Stempels, H. C., Collier Cameron, A., Hebb, L., Smalley, B., \& Frandsen, S. 2007, MNRAS, 379, 773

Szentgyorgyi, A. H., et al. 2005, BAAS, 37, 1339

Tegmark, M., et al. 2004, Phys. Rev. D, 69, 103501

Torres, G., Winn, J. N., \& Holman, M. J. 2008, ApJ, 677, 1324

Triaud, A. H. M. J., et al. 2010, A\&A, 524, A25

Valenti, J. A., \& Fischer, D. A. 2005, ApJS, 159, 141

Valenti, J. A., \& Piskunov, N. 1996, A\&AS, 118, 595

Vogt, S. S., et al. 1994, Proc. SPIE, 2198, 362

Wheatley, P. J., et al. 2010, arXiv:1004.0836

Winn, J. N., Fabrycky, D., Albrecht, S., \& Johnson, J. A. 2010, ApJ, 718, L145

Winn, J. N., Johnson, J. A., Albrecht, S., Howard, A. W., Marcy, G. W., Crossfield, I. J., \& Holman, M. J. 2009, ApJ, 703, L99

Winn, J. N., et al. 2005, ApJ, 631, 1215

Winn, J. N., et al. 2008, ApJ, 682, 1283 